improved but C-reactive protein (CRP) remained in the same levels. On demand anakinra prevented progression of prodromes to full-blown attacks which was demonstrated by decrease in the rate of attack/prodrome ratio $(p=0.002)$. On demand anakinra can be continued in 10 subjects on long-term but continuous treatment was required in 5 subjects.

Abstract THU0620 - Table 1. Comparison of attack characteristics before and after on demand anakinra protocol

\begin{tabular}{lccc}
\hline & $\begin{array}{c}\text { Colchicine } \\
\text { period }\end{array}$ & $\begin{array}{c}\text { Colchicine plus } \\
\text { On-Demand Anakinra } \\
\text { period }\end{array}$ & $\mathrm{p}$ \\
\hline Attack severity, VAS & $10(2)$ & $6(3)$ & 0.002 \\
Duration, days & $3(2)$ & $1.5(1)$ & 0.001 \\
Frequency*, number & $4(1.5)$ & $1.5(1.75)$ & 0.001 \\
CRP (mg/L) & $5.1(6.1)$ & $4.1(5.9)$ & 0.58 \\
AIDAI & $18(22.5)$ & $4(5)$ & 0.001 \\
Attack/prodrom ratio, & 1 & $0.6(0.4)$ & 0.002 \\
(n=10) & & $2(2.5)$ & 0.001 \\
Absenteeism, days & $7(8)$ & $2.5(3)$ & 0.002 \\
Presenteeism, days & $9(7.5)$ & &
\end{tabular}

Attack frequency and work productivity parameters are adjusted for 3 months intervals. VAS: visual analogue scale, CRP: C-reactive protein, AIDAI: autoinflammatory disease activity index

Conclusions: On demand anakinra significantly improved FMF attacks which suggest this approach would be of benefit in daily practice in selected patients.

Disclosure of Interest: None declared

DOI: 10.1136/annrheumdis-2018-eular.7198

\section{THU0621 $\quad$ PERSISTENT PRURITIC SKIN LESIONS WITH DYSKERATOTIC CELLS IN UPPER LAYER OF EPIDERMIS ARE SPECIFIC AND ASSOCIATED WITH HIGH LEVELS OF SERUM IL-18 IN ADULT-ONSET STILL'S DISEASE}

H. Nishikawa ${ }^{1}$, Y. Taniguchi ${ }^{1}$, N. Maeda-Aoyama ${ }^{1}$, K. Nakajima ${ }^{2}$, S. Inotani ${ }^{1}$ Y. Shimamura ${ }^{1}$, K. Inoue ${ }^{1}$, K. Arii ${ }^{3}$, S. Sano ${ }^{2}$, Y. Terada ${ }^{1} .{ }^{1}$ Nephrology and Rheumatology; ${ }^{2}$ Dermatology, Kochi Medical School Hospital, Nankoku; ${ }^{3}$ Internal Medicine, Kochi Red Cross Hospital, Kochi, Japan

Background: Adult-onset Still's disease (AOSD) is an acute and systemic inflam matory disorder that is characterised by high spiking fever, evanescent rash, arthralgia/arthritis and hyperferritinemia. However, recent reports showed that not only typical evanescent salmon-coloured rash but also atypical skin lesions, persistent pruritic papules and plaques, could be associated with AOSD.

Objectives: To assess the clinical significance of dyskeratotic cells (DCs) in skin lesions of AOSD.

Methods: We assessed histology of skin lesions including persistent pruritic skin lesions in Japanese patients with AOSD $(n=15)$. Moreover, we compared histology of AOSD with dermatomyositis (DM) $(n=6)$, drug eruptions (DE) $(n=7)$, and graft versus host disease (GVHD) ( $n=6)$.

Results: AOSD with persistent pruritic skin lesions $(n=10)$ histologically showed DCs only in upper layer of epidermis and horny layer without inflammatory cells infiltrations, indicating dyskeratosis. AOSD with evanescent rash $(n=5)$ histologically showed no DCs. DCs were positive by ssDNA staining, suggesting apoptotic cells. Serum IL-18 showed significantly higher in AOSD patients with dyskeratosis $(n=10)$ than without dyskeratosis $(n=5)$. In contrast to AOSD with DCs, the histology of DM, DE and GVHD demonstrated that DCs existed in all layers of epidermis with inflammatory cells infiltrations.

Conclusions: Persistent pruritic skin lesions in AOSD are specific by prominent epidermal apoptosis involving the upper layers of epidermis. Moreover, hyper IL18 might be related with dyskeratosis.

Disclosure of Interest: None declared

DOI: 10.1136/annrheumdis-2018-eular.3391

\section{THU0622 HISTOPATHOLOGY AND EXPRESSIONS OF CHEMOKINES, CXCL10, CXCL13, AND CXCR3, AND AN ENDOGENOUS LIGAND S100A8/A9 IN LYMPH NODES OF PATIENTS WITH ADULT-ONSET STILL'S DISEASE}

H.-A. Kim ${ }^{1}$, J.H. Han ${ }^{2} .{ }^{1}$ Department of Rheumatology; ${ }^{2}$ Department of Pathology, Ajou University School of Medicine, Suwon, Korea, Republic of Ireland

Background: Adult-onset still's disease (AOSD) is a rare systemic inflammatory disease with several symptoms, such as a persistent high spiking fever, typical rash, and lymphadenopathy. Endogenous factors related to interleukin (IL)-1, such as S100A8/A9 and several chemokines including CXCL10, CXCL13 and CXCR3, could play a potential role in the pathogenesis of AOSD.

Objectives: We aimed to find out typical histopathologic features, expressed pattern of chemokines in lymph nodes (LN) of AOSD patients.

Methods: Formalin-fixed paraffin-embedded excisional $L N$ tissues from 48 AOSD patients and 6 nonspecific reactive hyperplasia were histologically reviewed. The immunohistochemical stain for CXCL10, CXCL13, CXCR3 and S100A8/A9 were done. The clinical and laboratory data of the patients who underwent LN biopsies were reviewed.

Results: The LN specimens were categorised according to four distinctive patterns: follicular $(n=2,4.2 \%)$, paracortical $(n=19,39.6 \%)$, diffuse $(n=9,18.8 \%)$ hyperplasia, or mixed pattern $(n=18,37.5 \%)$. The other examined histologic features were presence of necrosis, karyorrhexis, immunoblastic, histiocystic and vascular proliferation. Most of the cases were required to take into differentia diagnosis such as dermatopathic lymphadenitis $(n=16,33.3 \%)$, lymphoma $(n=11$, $22.9 \%$ ) and histiocytic necrotizing lymphadenitis $(n=9,18.8 \%)$. The expression of chemokines and S100A8/A9 were higher than that of nonspecific reactive hyperplasia. The expression of chemokines and S100A8/A9 were higher than that of nonspecific reactive hyperplasia.

Conclusions: Histopathologic findings of LN in AOSD patients are diverse enough to be included various differential diagnosis. Because the several chemokines and S100A8/A9 were more expressed in AOSD patients than those of reactive hyperplasia, they may serve as a pathogenesis of AOSD.

Disclosure of Interest: None declared

DOI: 10.1136/annrheumdis-2018-eular.6238

\section{THU0623 1 SERUM IGG4 LEVELS AT DIAGNOSIS CAN PREDICT THE OUTCOMES OF UNTREATED PATIENTS WITH} IGG4-RELATED DISEASE: A RETROSPECTIVE STUDY

I. Mizushima, N. Suzuki, M. Yoshida, A. Takeji, T. Matsunaga, T. Zoshima, S. Hara, K. Ito, H. Fujii, K. Yamada, M. Kawano. Division of Rheumatology, Kanazawa University Hospital, Kanazawa, Kanazawa, Japan

Background: IgG4-related disease (IgG4-RD) is a recently recognised systemic fibro-inflammatory disorder that can affect many organs. ${ }^{1}$ In IgG4-RD, spontaneous, or at least temporary, remissions without treatment have been reported, and watchful waiting may be appropriate in certain patients with asymptomatic and inactive disease. ${ }^{2}$ However, the outcomes of patients with IgG4-RD who do not undergo treatment are still unclear.

Objectives: This study aimed to clarify the outcomes of untreated patients with IgG4-RD and the factors related to the outcomes.

Methods: We retrospectively reviewed the medical records of 107 patients with IgG4-RD, who were followed up for more than 6 months, at a single centre in Japan. Among them, 27 patients were followed up without treatment after the initial diagnosis. We compared the clinical features of these 27 patients with those of the 80 patients who underwent treatment. The outcomes of untreated patients were investigated, and logistic regression analysis was performed to assess factors related to the outcomes. Deterioration of IgG4-RD was defined as symptomatic, radiological, or functional exacerbation of the organ involved or new organ involvement.

Results: The patients comprised 73 men and 34 women (mean age 65.7 years) The follow-up periods were 7-252 (mean, 64.1) months, and the serum IgG4 lev els at diagnosis were 10.7-3610 (mean, 706) $\mathrm{mg} / \mathrm{dL}$. The 27 untreated patients had significantly fewer affected organs $(1.9 \pm 1.2$ vs $3.0 \pm 1.6, p=0.001)$, lower IgG4-RD responder index (10.8 \pm 5.1 vs $13.8 \pm 6.8, p=0.048)$, and lower frequency of ophthalmic and renal parenchymal lesions $(25.9 \%$ vs $53.8 \%, p=0.015$, and $3.7 \%$ vs $26.3 \%, p=0.012$, respectively) than did the 80 patients who underwent treatment. Of these 27 patients, 8 experienced deterioration of IgG4-RD 3-232 months (mean, 62.8) after the diagnosis. New organ involvement was observed in all 8 patients, 2 of whom concurrently suffered exacerbation of the organs involved. In age- and sex-adjusted logistic regression analysis, serum IgG4 elevation (per $100 \mathrm{mg} / \mathrm{dL}$, odds ratio 1.194, 95\% confidence interval 1.017-1.402 $\mathrm{p}=0.030$ ) was the only significant factor related to deterioration of disease in untreated patients with IgG4-RD.

Conclusions: The present study suggests that serum IgG4 levels may be useful to predict the outcomes of untreated patients with IgG4-RD, who tend to have fewer affected organs and lower lgG4-RD responder index.

\section{REFERENCES:}

[1] Stone JH, et al. IgG4-related disease. N Engl J Med 2012 Feb 9;366 (6):539-51.

[2] Khosroshahi A et al. International Consensus Guidance Statement on the Management and Treatment of IgG4-Related Disease. Arthritis Rheumatol 2015 Jul;67(7):1688-99.

Disclosure of Interest: None declared 